\title{
$11 \beta$-Hydroxysteroid Dehydrogenase 1 Human Tissue Distribution, Selective Inhibitor, and Role in Doxorubicin Metabolism ${ }^{\circledR}$
}

\author{
Xin Yang, Wenyi Hua, Sangwoo Ryu, Phillip Yates, Cheng Chang, Hui Zhang, and Li Di \\ Pharmacokinetics, Dynamics and Metabolism (X.Y., W.H., S.R., H.Z., L.D.) and Clinical Pharmacology (C.C.), Pfizer Inc., Groton, \\ Connecticut; and Early Clinical Development, Pfizer Inc., Cambridge, Massachusetts (P.Y.)
}

Received February 21, 2018; accepted April 16, 2018

\section{ABSTRACT}

$11 \beta$-Hydroxysteroid dehydrogenase $1(11 \beta$-HSD1) is distributed mainly in the human liver, with no detectable levels in the intestine or kidney, based on a newly developed proteomic approach. 11 $\beta$ HSD1 is mostly membrane-bound and retained in the liver microsomal fraction. Interindividual variability of $11 \beta$-HSD1 is relatively low, with about a 3-fold difference. A significant correlation was not observed between various demographic variables (ethnicity, gender, age, weight, smoking, and alcohol use) and 11 $\beta$-HSD1 protein expression or activity based on data from 31 donors. PF-915275 has been identified as a selective 11 $\beta$-HSD1 inhibitor with minimal effects on carbonyl reductase 1 and major cytochrome P450 enzymes. 11 $\beta$-HSD1 has been shown, for the first time, to be involved in doxorubicin metabolism, accounting for approximately $30 \%$ of doxorubicinol formation in human hepatocytes.

\section{Introduction}

$11 \beta$-hydroxysteroid dehydrogenase 1 (11 $\beta$-HSD1) belongs to the short-chain dehydrogenase/reductase superfamily. It is the only well known microsomal enzyme that plays a significant role in metabolizing drugs containing carbonyls. $11 \beta$-HSD1 acts as an NADPH-dependent reductase in intact cells but is a bidirectional enzyme capable of both reductase (reduction) and dehydrogenase (oxidation) reactions in microsomes, depending on the presence of the appropriate cofactors (NADPH for reduction or $\mathrm{NADP}^{+}$for oxidation, Fig. 1). The difference between $11 \beta$-HSD1 activity in intact cells and microsomes is likely owing to the accessibility of relevant cofactors to the enzyme. $11 \beta$-HSD1 has a pivotal physiologic role in transforming cortisone to cortisol (a stress hormone) that subsequently activates glucocorticoid receptors (Fig. 1). 11 $\beta$-HSD2, on the other hand, is a unidirectional dehydrogenase that converts active cortisol to inactive cortisone. $11 \beta$-HSD1 is a glycosylated protein that anchors at the luminal side of the endoplasmic reticulum via an $\mathrm{N}$-terminal transmembrane domain. As NADPH is impermeable through the endoplasmic reticulum membrane, colocalization and interaction between $11 \beta$-HSD1 and hexose-6-phosphate dehydrogenase provide a direct supply of NADPH for the reductase activity (Dzyakanchuk et al., 2009). $11 \beta$-HSD1 is involved in the metabolism of numerous clinically important drugs, such as benfluron, bupropion, ketoprofen, metyrapone, oracin, prednisolone, and triadimefon, as well as certain toxicants (e.g., nicotine-derived nitrosamine ketone) (Molnari and Myers, 2012; Skarydova and Wsol, 2012; Malatkova and Wsol, 2014). Selective inhibitors of $11 \beta$-HSD1 are promising agents for the treatment of type 2 diabetes and cardiovascular disease (Wang, 2006; Hale and Wang, 2008;

https://doi.org/10.1124/dmd.118.081083.

S This article has supplemental material available at dmd.aspetjournals.org.
Anderson and Walker, 2013). The X-ray single-crystal structure of $11 \beta$ HSD1 has been solved, which aids the structure-based design of potent and selective inhibitors (Hosfield et al., 2005; Julian et al., 2008). 11 $\beta$-HSD1 is expressed mainly in the liver but is also found in female reproductive tissues such as the placenta and ovaries (http://www.proteinatlas.org/). Studies have shown the upregulation of $11 \beta$-HSD1 in the pharyngeal mucosa and placentas of smokers, but the observed increase might be too small to lead to functional impact (Gronau et al., 2002; Huuskonen et al., 2008; Malatkova and Wsol, 2014). Since $11 \beta$-HSD1 has significant functional overlap with numerous cytosolic reductases, such as carbonyl reductase 1 (CBR1) and aldo-keto reductases (AKRs), protein abundance data can provide useful insights on its contribution to clearance relative to other reductases. In this study, a proteomic approach has been developed and applied to quantify $11 \beta$-HSD1 in human tissues for major clearance organs, including liver, intestine, and kidney, to understand more completely the tissue distribution of the enzyme at the protein level and its impact on drug clearance. To the best of our knowledge, this is the first proteomic approach to quantify $11 \beta$-HSD 1 at the protein level in human tissues. Interindividual variability was also evaluated using human liver microsomes (HLMs) from 31 donors with diverse histories of tobacco and alcohol use to examine the potential for demographic effects on $11 \beta$-HSD1 expression and activity. Relative expression factor (REF) and relative activity factor (RAF) were established to predict the contribution of $11 \beta$ HSD1 to clearance using human recombinant $11 \beta$-HSD1 (hr-11 $\beta$-HSD1). The $11 \beta$-HSD1 protein quantification data and tissue distribution information can help understand hepatic and nonhepatic contribution of the enzyme and to develop the physiologic-based pharmacokinetic models for $11 \beta$-HSD1-mediated clearance. The involvement of $11 \beta$ HSD1 to doxorubicin (Adriamycin) metabolism was investigated. Doxorubicin is one of the most cost-effective and widely used anticancer drugs (Hofman et al., 2015). The cardiotoxic metabolite doxorubicinol is

ABBREVIATIONS: AKR, aldo-keto reductase; CBR1, carbonyl reductase 1; ER, endoplasmic reticulum; HHEP, human hepatocyte; HLC, human liver cytosol; HLM, human liver microsome; hr-11 $\beta$-HSD1, human recombinant $11 \beta$-hydroxysteroid dehydrogenase 1 ; $11 \beta$-HSD1, $11 \beta$ hydroxysteroid dehydrogenase $1 ; 11 \beta$-HSD2, $11 \beta$-hydroxysteroid dehydrogenase 2; IDA, information-dependent acquisition; IS, internal standard; LC, liquid chromatography; LC-MS/MS, liquid chromatographic-tandem mass spectrometry; MS, mass spectrometry; P450, cytochrome P450; RAF, relative activity factor; REF, relative expression factor; SRM, selected reaction monitoring. 


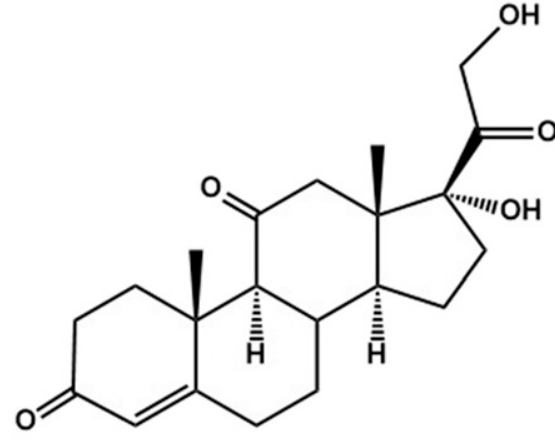

Cortisone (Inactive)

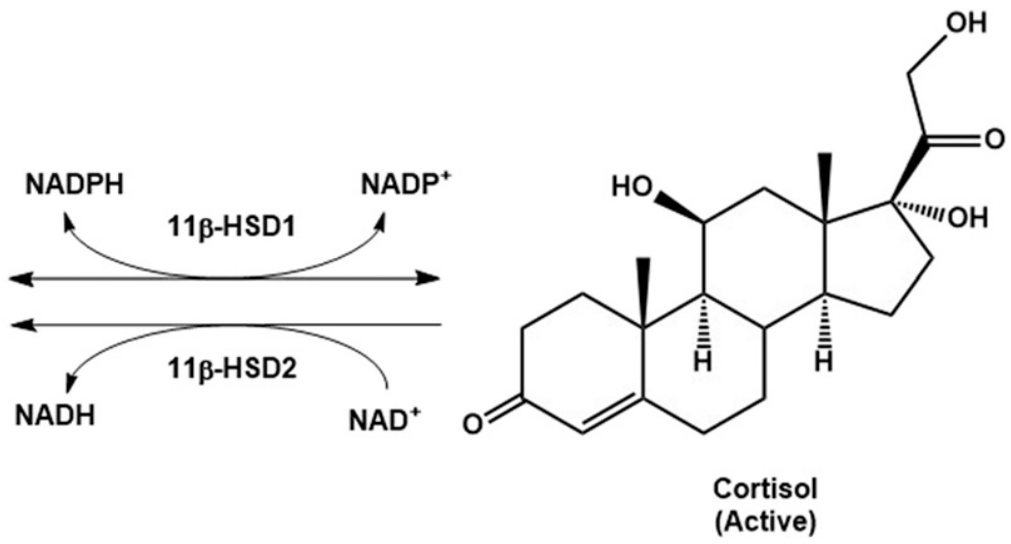

Fig. 1. Interconversion between cortisone and cortisol catalyzed by $11 \beta$-HSD1 and $11 \beta$-HSD2. In intact cells, $11 \beta$-HSD1 metabolizes cortisone to cortisol using NADPH and generates $\mathrm{NADP}^{+}$. In microsomes, $11 \beta$-HSD1 catalyzes the bidirectional reaction, depending on the cofactors available. In the presence of NADPH, $11 \beta$-HSD1 reduces cortisone to cortisol. In the presence of $\mathrm{NADP}^{+}, 11 \beta$-HSD1 oxidizes cortisol to cortisone. $11 \beta$-HSD2 is a unidirectional enzyme that oxidizes cortisol to cortisone using $\mathrm{NAD}^{+}$cofactor and generates NADH.

thought to be formed mostly by CBR1, with minor contributions from AKRs (Kassner et al., 2008). In this study, we demonstrate, for the first time, the role of $11 \beta$-HSD1 in metabolizing doxorubicin to generate the metabolite doxorubicinol.

\section{Materials and Methods}

\section{Materials}

Pools of 3-12 donors (both male and female) of human intestine (prepared from duodenal and jejunal tissues), kidney cytosols and microsomes, and HLMs of 31 individual donors were obtained from Xenotech (Kansas City, KS). The pooled HLMs (50 donors of both males and females) and cynomolgus monkey intestine microsomes (338 donors of both males and females, used as a control matrix) were purchased from Corning (Tewksbury, MA). Human liver cytosols (HLCs, 16 donors of both males and females) and cryopreserved human hepatocytes (lot no. DCM, 10 donors) were from BioreclamationIVT (Baltimore, MD). We obtained hr-11 $\beta$-HSD1 from OriGene Technologies (catalog no. TP312093, lot no. 021617, Rockville, MD) and Cayman Chemical (catalog no. 10007815, lot 0486950, Ann Arbor, MI). Stable isotope-labeled (purity $>95 \%$ and isotopic purity $>99 \%$ ) peptides used as internal standards (IS) and standard peptides were purchased from New England Peptide (Gardner, MA). Peptide purity was determined by the vendor using high-performance liquid chromatography-UV and identified by mass spectrometry (MS) analysis. Amino acid analysis was also performed by the vendor to determine net peptide content. Concentrations of synthesized peptides were corrected by purity data for quantitation. Rapigest SF was from Waters (Milford, MA), and MS-grade trypsin was purchased from Thermo Scientific (Guilford, CT). Cortisone, cortisol, doxorubicin, doxorubicinol, and PF-915275 (CAS no. 857290-04-1, N-(6-amino-2-pyridinyl)-4'-cyano[1,1'biphenyl]-4-sulfonamide) (Siu et al., 2009) were from Pfizer Global Material Management (Groton, CT). Iodoacetamide, dithiothretol, and other chemicals were from Sigma-Aldrich (St. Louis, MO) unless specified otherwise.

\section{Peptide Mapping and Selection}

Sample Preparation for Peptide Mapping and Selection. Peptide mapping and selection experiments were initially performed with $0.4 \mathrm{mg}$ of protein of pooled HLMs and hr-11 $\beta$-HSD1 protein standards. The samples were prepared using methods described previously (Hua et al., 2017). Briefly, the samples were mixed with 4 volumes of $50 \mathrm{mM}$ ammonium bicarbonate buffer $(\mathrm{pH} 8.5)$ containing $0.1 \%$ Rapigest $\mathrm{SF}$ in a 1-ml 96-well LoBind plate (Eppendorf, Hauppauge, NY) and heated at $\sim 80^{\circ} \mathrm{C}$ for 5 minutes in a water bath (VWR, Radnor, PA). The proteins were reduced by $5 \mathrm{mM}$ dithiothreitol at $37^{\circ} \mathrm{C}$ for 30 minutes, followed by incubation with $10 \mathrm{mM}$ iodoacetamide for 30 minutes in the dark. The final solution was incubated with trypsin at 20:1 (protein/trypsin) ratio overnight at $37^{\circ} \mathrm{C}$ under mild agitation. The tryptic peptide solution was acidified to $\mathrm{pH}<2$ with formic acid and transferred to a clean 1-ml 96-well LoBind plate followed by brief centrifugation. Acetonitrile $(20 \mu \mathrm{l})$ was added to the samples, and the final organic solvent was $5 \%$.
LC-MS/MS Methods for Peptide Mapping and Selection. The liquid chromatographic-tandem mass spectrometry (LC-MS/MS) methods for peptide mapping using information-dependent acquisition (IDA) and SWATH are summarized in Supplemental Material (Tables S1 and S2). Briefly, $20 \mu \mathrm{l}$ of the digested samples was injected onto a BEH C18 column (XBridge $130 \AA$ A $100 \times$ $2.1 \mathrm{~mm}, 2.5 \mu \mathrm{m}$; Waters) by the CTC PAL autosampler (Leap Technologies, Carrboro, NC). A triple time-of-flight 6600 mass spectrometer (Sciex, Toronto, Canada) was used for data acquisition. An IDA experiment was first performed to provide peptide identification using ProteinPilot Software 5.0 (Sciex) with the Paragon database search algorithm (proteome library: Uniprot_Homo_sampiens, updated 2015/02). Both biologic modifications and amino acid substitutions were searched in "thorough" mode. The samples were then subjected to SWATH acquisition on the same LC-triple time-of-flight MS instrument. The resulting protein "pilot.group" file from IDA acquisition was used to generate the ion library to guide SWATH data processing. The digested samples were reinjected on an API 5500 (Sciex) triple quadrupole MS in selected- reaction monitoring (SRM) mode to evaluate the quantitation performance of the peptides. Five peptides VLGLIDTETAMK, AVSGIVHMQAAPK, VIVTGASK, EYSVSR, and FALDGFFSSIR (abbreviated as VLG, AVS, VIV, EYS, and FAL hereafter) were initially selected as probe peptides. Selection criteria for surrogate peptides were similar to those described previously (Balogh et al., 2013). High amino acid sequence coverage was achieved for hr-11 $\beta$-HSD1 (81\%) and HLMs (53\%), with a confidence level cutoff $>95 \%$. The light and isotopic stable-labeled peptide standards were synthesized (Table 1) and used for further evaluation of stability, measurement accuracy, and digestion efficiency. Final liquid chromatography (LC) separation and SRM conditions on triple quadrupole MS were fine-tuned with synthesized standards and are summarized in Supplemental Material (Table S3) and Table 1.

\section{Evaluation of Peptides and Optimization of Digestion Efficiency}

Accuracy and Stability Evaluation. The stability of the five selected peptides was evaluated in the presence and absence of HLMs. In the stability experiments without HLMs, $20 \mu \mathrm{l}$ of combined peptide solution ( $1 \mu \mathrm{M}$ each) in four replicates was used instead of HLMs and incubated for 3, 5, 9, and 16 hours using the procedure described in the Sample Preparation for Peptide Mapping and Selection section. To evaluate peptide stability in HLMs, the HLM $(0.4 \mathrm{mg}$ of total protein) was spiked with $5 \mu \mathrm{l}$ of combined peptide solution $(16 \mu \mathrm{M}$ each of the five peptides) in four replicates and incubated using the same procedure as described above for 3, 5, 9, and 16 hours. HLM ( $0.4 \mathrm{mg}$ of protein) samples without peptides were also processed in parallel and used as baseline. Calibration curves were prepared by spiking combined peptide solution into digested control matrix (cynomolgus monkey intestine microsomes). Calibration curve preparation details are described in the Sample Preparation for 11 $\beta$-HSD1 Quantitation section. Quantification was performed against peptide calibration curves. Measurement accuracy for the spiked peptides was calculated by dividing the concentrations of the peptides in the spiked samples after subtracting the baseline by the nominal concentrations. 
TABLE 1

MS method for peptide analysis using SRM acquisition

\begin{tabular}{|c|c|c|}
\hline Mass Spectrometer & \multicolumn{2}{|c|}{ Sciex API-5500-Electrospray (+) } \\
\hline $\begin{array}{l}\text { Data collection software/version } \\
\text { Ion source temperature } \\
\text { IonSpray voltage } \\
\text { Declustering potential } \\
\text { Dwell time } \\
\text { SRM transitions (and collisional } \\
\text { energy settings) }\end{array}$ & 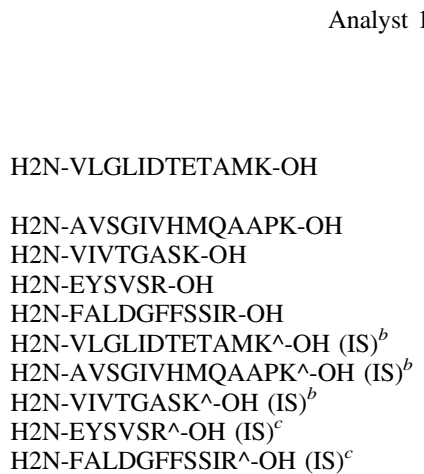 & 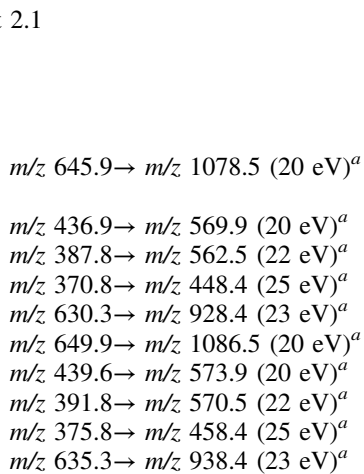 \\
\hline
\end{tabular}

${ }^{a}$ Collision energy.

${ }^{b} \mathrm{~K}^{\wedge}$-IS peptides were labeled at C-terminal arginine $(\mathrm{K})=\left({ }^{13} \mathrm{C}\right)_{6} \mathrm{H}_{14}\left({ }^{15} \mathrm{~N}\right)_{2} \mathrm{O}_{2}$ (mass shift +8 ).

${ }^{c} \mathrm{R}^{\wedge}$-IS peptides were labeled at $\mathrm{C}$-terminal arginine $(\mathrm{R})=\left({ }^{13} \mathrm{C}_{6} \mathrm{H}_{14}\left({ }^{15} \mathrm{~N}\right)_{4} \mathrm{O}_{2}\right.$ (mass shift +10 )

Since the final samples were acidified to below $\mathrm{pH} 2$ after digestion and there is a wait time before injection for LC-MS/MS analysis, peptide stability was evaluated using these conditions. The digested control matrix was spiked with $10 \mu \mathrm{l}$ of $1 \mu \mathrm{M}$ combined solution of five peptides and acidified with formic acid to $\mathrm{pH}<2$. The solution was stored at room temperature in an autosampler and sampled at $0,9,30$, and 34 hours in triplicate. The combined solution $(10 \mu \mathrm{l})$ of five isotope-labeled peptides at $0.25 \mu \mathrm{M}$ each was added to the samples before injection and used as IS. Peak area ratio between analyte and IS was used for stability evaluation.

Digestion Efficiency. To obtain optimal trypsin digestion efficiency, the digestion time was evaluated with pooled HLMs $(0.4 \mathrm{mg}$ protein $)$ in four replicates using the same procedure as described in the Sample Preparation for Peptide Mapping and Selection section for 3, 5, 9, and 16 hours. Calibration curves were prepared by spiking combined peptide solution into the digested control matrix. The details of preparing calibration curves are described in the section Sample Preparation for 11 $\beta$-HSD1 Quantitation. Results (picomoles per milligram protein) of individual peptides were calculated using the standard curves.

\section{Sample Preparation for 11 $\beta$-HSD1 Quantitation}

For quantitation, samples containing $0.4 \mathrm{mg}$ of protein were processed in triplicate using the same procedure as described in Sample Preparation for Peptide Mapping and Selection. The samples were tryptic digested for 9 hours. Pooled monkey intestine microsomes were used as control matrix for calibration curves. Stock solutions $(200 \mu \mathrm{M})$ of standard peptides and stable isotope-labeled peptides were individually prepared in dimethylformamide. For calibration curves, the combined standard solutions of the peptides were prepared in the range of $0.010-30 \mu \mathrm{M}$ in 1:1 dimethylformamide/water. Ten microliters of calibration standards were spiked into the digested control matrix, and $10 \mu \mathrm{l}$ of the combined IS at $0.25 \mu \mathrm{M}$ in the same diluent were spiked into all the digested samples (except double blanks) after acidification. The standard curve quantitation range is equivalent to $0.25-750 \mathrm{pmol} / \mathrm{mg}$ protein, assuming complete digestion of $11 \beta$-HSD1 in the sample matrix.

\section{LC-MS/MS SRM Sample Analysis}

LC triple-quadrupole MS in SRM mode was used to optimize digestion efficiency, evaluate peptide stability, and determine final $11 \beta$-HSD1 concentration in the sample matrix. Autosampler and LC-MS/MS methods are summarized in Supplemental Material (Table S3) and Table 1. Analysis of the SRM data were performed using MultiQuant Software 2.1 (Sciex).

\section{Measurement of 11 $\beta$-HSD1 Activity in Different Matrices}

Cortisone-to-cortisol formation was used as an $11 \beta$-HSD1-specific reaction to evaluate enzyme activity in human intestine, kidney, and liver microsomes $(0.75 \mathrm{mg} / \mathrm{ml})$ and cytosols $(1 \mathrm{mg} / \mathrm{ml})$ and $\mathrm{hr}-11 \beta$-HSD1 $(10 \mu \mathrm{g} / \mathrm{ml})$ at $1 \mu \mathrm{M}$. The final dimethylsulfoxide concentration was $0.03 \%$, and the NADPH cofactor was $1.4 \mathrm{mM}$. The solution was incubated at $37^{\circ} \mathrm{C}$ for 1 hour in a heating block (Boekel Scientific, Feasterville-Trevose, PA). At appropriate time points, an aliquot of solution was removed and quenched with cold acetonitrile containing IS. Cortisol metabolite formation was quantified using LC-MS/MS via a standard curve. Intrinsic clearance was calculated based on the rate of cortisol metabolite formation. Duplicates or triplicates were used for each study. The LC mobile phases were $0.1 \%$ formic acid in water (phase a) and $0.1 \%$ formic acid in acetonitrile (phase b). The solvent gradient was $95 \%$ (a)/5\% (b) for 0.3 minute, $5 \%$ (a)/95\% (b) from 0.3 to 1.30 minutes and held 2.3 minutes, $95 \%$ (a)/5\% (b) from 2.3 to 2.31 minutes. The compounds were eluted from the column (Acquity C18, $1.7 \mu \mathrm{m}, 2.1 \mathrm{~mm} \times 50 \mathrm{~mm}$; Waters) at a flow rate of $0.5 \mathrm{ml} / \mathrm{min}$. Samples $(10 \mu \mathrm{l})$ were injected using a CTC PAL autosampler (Leap Technologies, San Diego, CA) for analysis. Shimadzu high-performance liquid chromatography AD30 pumps (Columbia, MD) connected to an AB Sciex (Foster City, CA) 5500 triplequadrupole mass spectrometer equipped with a TurbolonSpray source were used. Analyst 1.6.2 software (Applied Biosystems, Foster City, CA) was used for data collection, processing, and analysis. The IS for LC-MS/MS quantification was terfenadine. The MS conditions at positive- ion SRM mode are cortisol Q1 363.2, Q3 121.2, DP 60, CE 60 and terfenadine Q1 472.2, Q3 436.3, DP 60, CE 60.

\section{Evaluation of the Role of 11 $\beta$-HSD1 in Doxorubicin Metabolism}

Doxorubicin $(100 \mu \mathrm{M})$ was incubated with $\mathrm{hr}-11 \beta$-HSD1 $(100 \mu \mathrm{g} / \mathrm{ml}$, from both Cayman Chemical and OriGene Technologies), with a final dimethylsulfoxide concentration of $1 \%$ and NADPH cofactor of $1.4 \mathrm{mM}$ at $37^{\circ} \mathrm{C}$ for 1 hour in a heating block in duplicate. Samples without the addition of NADPH were used as negative controls. At appropriate time points, an aliquot of solution was removed and quenched with cold acetonitrile containing IS. Doxorubicinol reduction metabolite formation was quantified using LC-MS/MS in positive-ion SRM mode with Q1 546, Q3 363, DP 60, and CE 30.

\section{Evaluation of Concentration and Selectivity for 11 $\beta$-HSD1 Inhibitor PF-915275}

The inhibitory concentration of $11 \beta$-HSD1 inhibitor PF-915275 was determined in cryopreserved human hepatocytes at 0.5 million cells/milliliter using cortisone as a substrate $(1 \mu \mathrm{M})$ and via monitoring cortisol formation (see preceding discussion for LC-MS/MS conditions). Detailed protocols on cytochrome P450 (P450) selectivity determination have been reported previously (Yang et al., 2016). Briefly, human hepatocytes ( 0.5 million cells/milliliter) were incubated with PF-915275 at $1 \mu \mathrm{M}$ in the presence of probe substrates at $1 \mu \mathrm{M}$. The following specific substrate reactions were monitored with and without $11 \beta$ HSD1 inhibitor PF-915275 at $1 \mu \mathrm{M}$ to evaluate the selectivity against several P450s: phenacetin (1A2) to acetaminophen, bupropion (2B6) to OH-bupropion, 
paclitaxel (2C8) to $6 \alpha-\mathrm{OH}$-paclitaxel, diclofenac (2C9) to 4 '-OH-diclofenac, S-mephenytoin (2C19) to $4^{\prime}-\mathrm{OH}-\mathrm{S}$-mephenytoin, dextromethorphan (2D6) to dextrorphan, and midazolam (3A) to 1'-OH-midazolam. Metabolite formation was monitored over 1 hour and used to calculate the percentage of inhibition of the enzymes. Selectivity of PF-915275 $(1 \mu \mathrm{M})$ against CBR1 was conducted using doxorubicin as a substrate $(1 \mu \mathrm{M})$ in hr-CBR1 (human recombinant CBR1, $10 \mu \mathrm{g} / \mathrm{ml}$ ) and monitored for the formation of doxorubicinol over a 1-hour incubation. Because a specific CRB1 substrate reaction is currently not available, hr-CBR1 was used to evaluate PF-915275 selectivity against CBR1.

\section{Determination of 11 $\beta$-HSD1 Contribution to Doxorubicin Metabolism}

Doxorubicin $(1 \mu \mathrm{M})$ was incubated with cryopreserved human hepatocytes at 0.5 or 2 million cells/milliliter with and without the presence of $11 \beta$-HSD1 inhibitor PF-915275 at $1 \mu \mathrm{M}$. The incubation was done at $37^{\circ} \mathrm{C}$ in a carbon dioxide incubator $\left(5 \% \mathrm{CO}_{2} / 95 \%\right.$ air) with $75 \%$ relative humidity on an orbital shaker (VWR) at $150 \mathrm{rpm}$ for 4 hours. At various time points, samples were taken and quenched with cold acetonitrile containing IS. The solution underwent centrifugation, and the supernatant was transferred to a clean plate and injected to LC-MS/MS for analysis. Doxorubicinol metabolite formation was used to evaluate the contribution of $11 \beta$-HSD1 to doxorubicin reduction (see preceding for MS conditions). Percent inhibition was calculated using the initial slope of the doxorubicinol metabolite formation curve with and without the inhibitor. The studies of $11 \beta$-HSD1 contribution to doxorubicin reduction were performed on five different days in duplicate, and the average of all the data were reported

\section{Results}

This is the first study reporting $11 \beta$-HSD1 protein expression in human intestine, kidney, and liver using a newly developed proteomic approach. The selection criteria for the surrogate peptides for protein quantification include that they are unique within the human proteome, have 6-20 amino acid residues, and have no known post-translational modifications, single nucleotide polymorphisms, labile amino acids, or missed cleavage sites. Five surrogate peptides meeting the criteria were selected for further evaluation of stability and digestion efficiency (Table 1). Peptide amounts (in picomoles per milligram of protein) at different time points were quantitated using calibration curves to evaluate stability and digestion efficiency. It is important to use the quantified amount instead of the MS response for these evaluations as the MS response indicates only the relative stability or completeness of digestion rather than the absolute amount. Peptide bonds can be cleaved with a wide range of rates during digestion, and the ability of enzymes to cleave different scissible bonds varies (Šlechtová et al., 2015), even when the reaction time is sufficient. The stability of the five selected peptides under tryptic digestion conditions were examined in the presence and absence of HLMs. The results showed that all five peptides were stable without HLMs for at least 16 hours with $90 \%-$ $110 \%$ remaining (Supplemental Material, Table S4). These data suggest that the peptides had good chemical stability and no significant observable loss. In the presence of HLMs, all the peptides had stability $\geq 87 \%$ at 9 hours with the exception of FLA (Supplemental Material, Table S5). FLA had 40\% remaining at 9 hours and was too unstable to be used as a surrogate peptide for quantitation.

Ideally, the concentration for each of the cleaved peptides should be the same after digestion is complete; however, because of different cleavage rates, accessibility of scissible bonds, chemical and physical peptide stability, and so forth, the peptide concentrations detected by LC-MS/MS after digestion are often different, and the peptides providing the highest concentration should be used as surrogate peptides for protein quantitation. For the digested samples of $11 \beta$-HSD1, the peptide concentrations for VLG, EYS, and FLA were significantly lower than for AVS and VIV (Fig. 2). VLG had the lowest digestion efficiency and was not suitable as a surrogate peptide even though it demonstrated good stability. FAL decreased significantly after 5 hours owing to instability. EYS concentration increased over the 16-hour digestion period. It offered attractive properties as a surrogate peptide for quantitation, but longer digestion times ( $>16$ hours) would be needed. AVS and VIV had the highest measured concentrations with comparable results at both 9 and 16 hours of digestion. Both demonstrated acceptable stability during the digestion process ( $\geq 87 \%$ remaining after 9 hours) and were therefore selected as surrogate peptides for quantitation. Overall, the 9-hour digestion period offered the maximum efficiency for the AVS and VIV peptides and was used for subsequent sample preparation (Fig. 2). Stability under autosampler storage conditions was also examined at room temperature in solution with $\mathrm{pH}<2$. AVS and VIV were both stable over 34 hours $(<5 \%$ change, data not shown), suggesting a lack of instability concerns for these peptides stored in the autosampler before analysis.

We measured $11 \beta$-HSD1 protein abundance in both cytosols and microsomes of human intestine, kidney, and liver using both AVS and VIV peptides (Table 2). The two surrogate peptides showed linear response over the concentration range of $0.25-750 \mathrm{pmol} / \mathrm{protein}$. In control matrix (cynomolgus monkey intestine microsomes), no interference peak was found for AVS, and the signals were less than $20 \%$ of the lower limit of quantitation for VIV. These data suggested that $11 \beta$-HSD1 was not present in human intestine and kidney, whereas high abundance was found in HLMs with $62 \mathrm{pmol} / \mathrm{mg}$ protein for a pooled lot of 50 donors. Only trace amounts were detected in liver cytosol (3.5 $\mathrm{pmol} / \mathrm{mg}$ protein), a result potentially due to contamination from the microsomal fraction during fractionation of the liver samples. For the three clearance organs examined, $11 \beta$-HSD1 appears to be expressed only in liver, with a significant amount as a membrane bound protein. $11 \beta$-HSD1 abundance in HLMs from 31 donors was also quantified to explore interindividual variability and demographic effects (Table 2). $11 \beta$-HSD1 protein amounts ranged from 36 to $109 \mathrm{pmol} / \mathrm{mg}$ protein, suggesting relatively small interindividual variability of approximately threefold. The demographic information (e.g., ethnicity, gender, age, weight, smoking, and alcohol use) of the individual liver donors is included in the Supplemental Material (Table S6). Using a variety of exploratory statistical analyses (e.g., $t$ test, one-way analysis of variance, both simple and multiple regressions), we did not obtain robust evidence of statistically significant effects between the demographic variables and $11 \beta$-HSD1 abundance or intrinsic clearance (results not shown). For $\mathrm{hr}-11 \beta$-HSD1, the amounts were 4161 and $5686 \mathrm{pmol} / \mathrm{mg}$ protein for the materials from OriGene Technologies and Cayman Chemical, respectively. The REF values based on both lots of hr-11 $\beta$-HSD1 are summarized in Table 3.

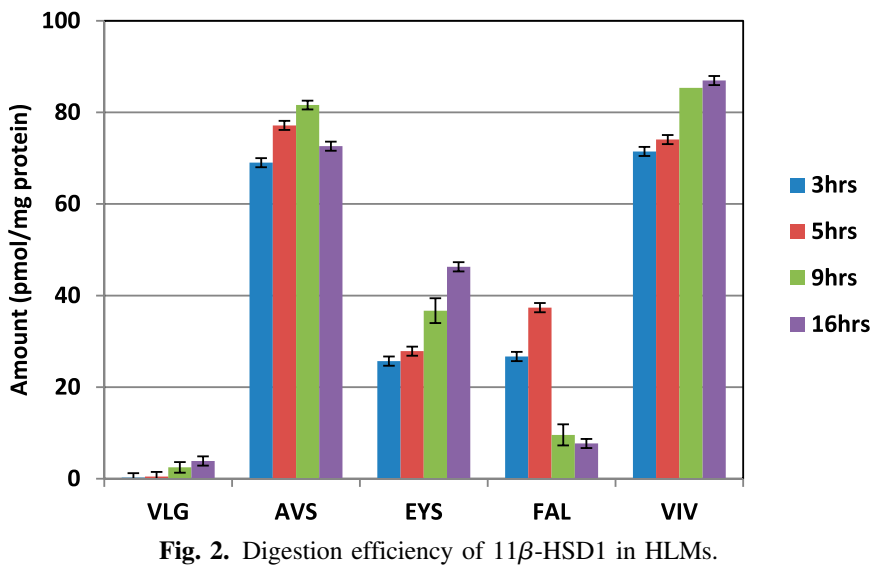


TABLE 2

$11 \beta$-HSD1 protein abundance in individual/pooled human liver, intestine (HIM), and kidney cytosols (HKCs) and microsomes $(n=3)$

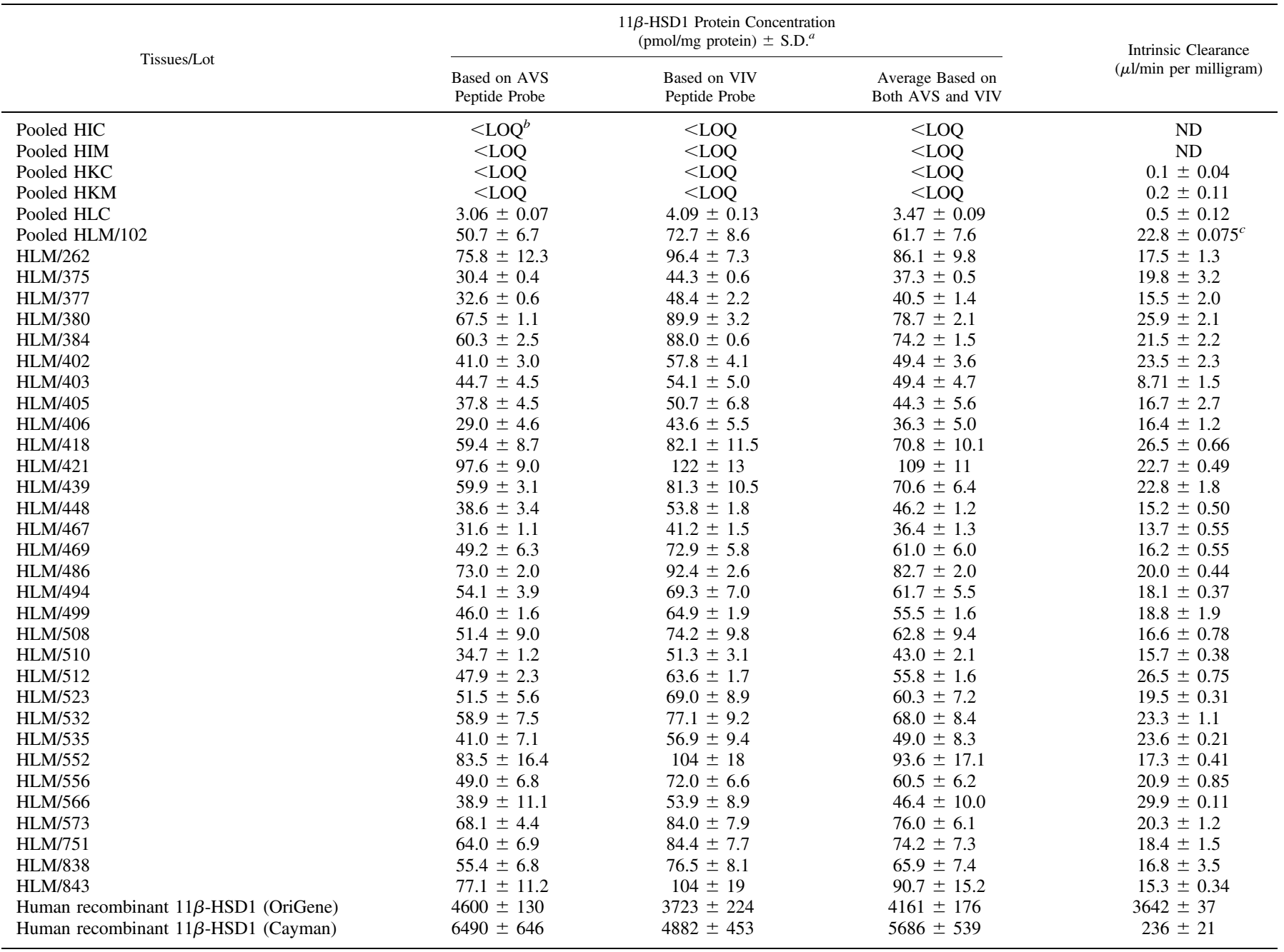

LOQ, lower limit of quantitation; ND, not determined.

${ }^{a}$ Data generated from three replicates of each sample.

${ }^{b} \mathrm{LOQ}$ is $0.25 \mathrm{pmol} / \mathrm{mg}$ protein.

${ }^{c}$ Intrinsic clearance in human hepatocytes is determined to be $6.2 \pm 0.22 \mu \mathrm{l} / \mathrm{min}$ per million cells or $15.6 \pm 0.55 \mathrm{ml} / \mathrm{min}$ per kilogram, a value comparable to scaled $\mathrm{HLM}$ data (i.e., $21.5 \mathrm{ml} / \mathrm{min}$ per kilogram).

We measured $11 \beta$-HSD1 catalytic activity using cortisone as a substrate and monitored for cortisol metabolite formation. The rate of cortisol formation is summarized in Table 2. The intrinsic clearance values range from 8.7 to $30 \mu 1 /$ min per milligram in pooled or individual HLM donors. The intrinsic clearance in human hepatocytes was also determined to be $6.2 \pm 0.22 \mu \mathrm{l} / \mathrm{min}$ per million cells or $15.6 \pm$ $0.55 \mathrm{ml} / \mathrm{min}$ per kilogram, an estimate comparable to the scaled HLM value in the pooled lot (HLM/102), that is, $21.5 \mathrm{ml} / \mathrm{min}$ per kilogram. The interindividual variability of $11 \beta$-HSD 1 based on functional activity of cortisone reduction is approximately 3 -fold, a relatively small value and in line with those based on protein expression. The $11 \beta$-HSD1 activity of the $\mathrm{hr}-11 \beta$-HSD1 is $3642 \mu \mathrm{l} / \mathrm{min}$ per milligram for the OriGene Technologies lot and $236 \mu \mathrm{l} / \mathrm{min}$ per milligram for the Cayman Chemical lot. Even though the protein levels are similar for both vendors, the $11 \beta$-HSD1 activity is 15 -fold higher for the material from OriGene Technologies relative to Cayman Chemical. The correlation between $11 \beta$-HSD1 protein abundance and activity was poor $\left(R^{2}=\right.$ 0.066 , Fig. 3A) when using HLM data only, a result potentially resulting from a narrow data range $(\sim 3$-fold $)$. With the addition of HLC and hr-11 $\beta$-HSD1 data, to increase the spread of these data over multiple log units, $11 \beta$-HSD1 protein abundance and activity now appear correlated $\left(R^{2}=0.82\right.$, Fig. 3B). The RAF values based on both lots of hr-11 $\beta$-HSD1, and both HLM and human hepatocyte (HHEP) are summarized in Table 3.

With our improved understanding of tissue distribution, the contribution of $11 \beta$-HSD 1 to the formation of the doxorubicinol metabolite from doxorubicin was investigated. Doxorubicinol metabolite formulation was reported in the literature to be mostly mediated by CBR1 with minor contribution from AKRs (Kassner et al., 2008). Incubation of

TABLE 3

RAF and REF values for human recombinant $11 \beta$-HSD1

\begin{tabular}{cccc}
\hline $\begin{array}{c}\text { Human Recombinant } \\
11 \beta \text {-HSD1 Vendor }\end{array}$ & $\begin{array}{c}\text { RAF Based } \\
\text { on HLMs }\end{array}$ & $\begin{array}{c}\text { RAF Based on } \\
\text { Human Hepatocytes }\end{array}$ & REF Based on HLMs \\
\hline OriGene, lot no. 021617 & 0.0063 & 0.0045 & 0.015 \\
Cayman, lot no. 0486950 & 0.097 & 0.070 & 0.011 \\
\hline
\end{tabular}




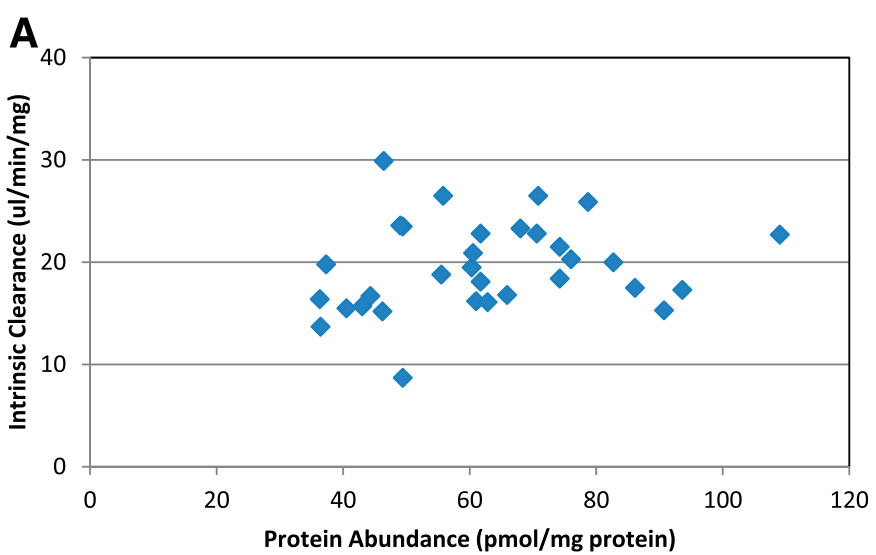

B

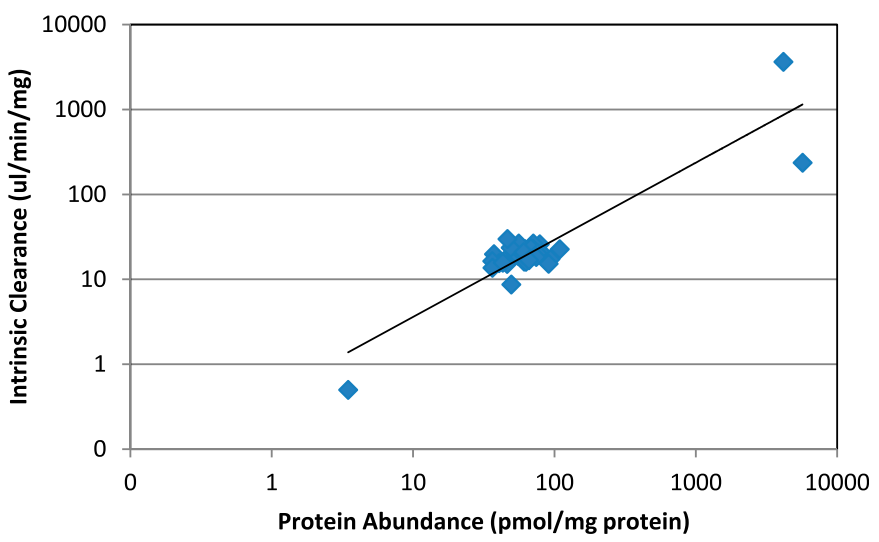

Fig. 3. Protein abundance and intrinsic clearance correlation for $11 \beta$-HSD1. (A) Data from HLMs only. (B) Data include HLMs, HLCs, and hr-11 $\beta$-HSD1 enzyme.

doxorubicin with hr-11 $\beta$-HSD1 from Cayman Chemical indicated doxorubicinol metabolite formation (Fig. 4). Formation of doxorubicinol metabolite was also observed using hr-11 $\beta$-HSD1 from OriGene Technology (data not shown), suggesting for the first time that $11 \beta$ HSD1 is involved in the metabolism of doxorubicin to the metabolite doxorubicinol. These results were further verified using HHEP by monitoring doxorubicinol metabolite formation with and without $11 \beta$ HSD1 inhibitor PF-915275. PF-915275 has been reported to be a highly potent inhibitor for $11 \beta$-HSD1 (Siu et al., 2009). The selectivity of PF-915275 against a variety of P450s and CBR1 was evaluated using HHEPs (Fig. 5). PF-915275 at $1 \mu \mathrm{M}$ was not only a potent inhibitor of $11 \beta$-HSD1 (89\%) but was also selective against CBR1 and the major $\mathrm{P} 450$ s, with only a minor inhibition of CYP2C19 (29\%). The contribution of $11 \beta$-HSD1 to doxorubicinol formation was $29 \% \pm$ $7.9 \%$ based on metabolite formation in HHEP with and without inhibitor PF-915275 (Table 4). Because doxorubicin does not have significant turnover in human hepatocytes, the intrinsic clearance is not measurable. Therefore, REF and RAF approaches and the metabolite formation rate were not applied to estimate the percent contribution to clearance by $11 \beta$-HSD1.

\section{Discussion}

This article is the first study to quantify $11 \beta$-HSD1 levels using a newly developed proteomic approach. Tissue distribution of $11 \beta$-HSD1 is predominately in the human liver microsomal fraction with a moderate concentration ( $60 \mathrm{pmol} / \mathrm{mg}$ protein $)$ similar to that of CYP2C9

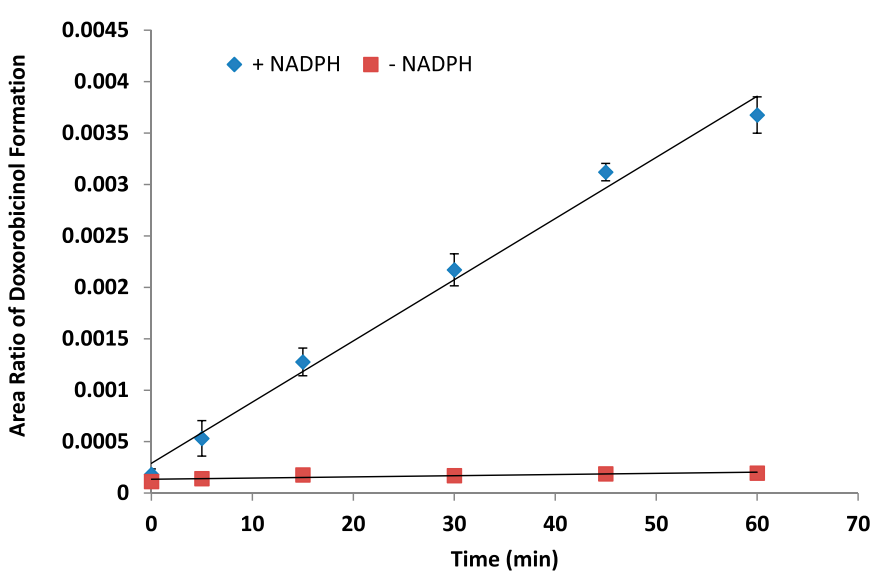

Fig. 4. Formation of doxorubicinol in hr-11 $\beta$-HSD1 with and without NADPH.

(72 $\mathrm{pmol} / \mathrm{mg}$ protein) in the liver (SIMCYP database). $11 \beta$-HSD1 was not detectable in the intestine or kidney, indicating the critical physiologic role for this enzyme in the liver for conversion of cortisone to cortisol. The interindividual variability of $11 \beta$-HSD1 was approximately 3 -fold based on both protein levels and enzymatic activity. This variability is quite small, suggesting that the enzyme is highly regulated, a hypothesis consistent with the important physiologic function of the enzyme in converting inactive cortisone to active cortisol, a stress hormone known to activate glucocorticoid receptors. Because of the relatively narrow protein distribution of $11 \beta$-HSD1 in the liver for the individual donors, the correlation between protein amount and enzyme activity was not observed; but, when the distribution range is increased by adding HLCs and hr-11 $\beta$-HSD1 data, a correlation was observed between protein expression and $11 \beta$-HSD1 activity. Various available demographic factors, such as ethnicity, gender, age, weight, smoking, and alcohol use, did not appear to impact $11 \beta$-HSD1 activity based on this study, although the sample size was relatively small and the sample demographic distribution was not evenly balanced or represented in the various groups. Tobacco use has been reported to upregulate $11 \beta$-HSD1 expression in the pharyngeal mucosa and placentas of smokers, although no functional changes have been observed (Gronau et al., 2002; Huuskonen et al., 2008; Malatkova and Wsol, 2014). The impact of smoking on liver $11 \beta$-HSD1 levels has not been reported. In this study, significant statistical differences were not observed between smokers and nonsmokers using either $11 \beta$-HSD 1 protein expression or activity. The protein quantification and activity data are useful to estimate the

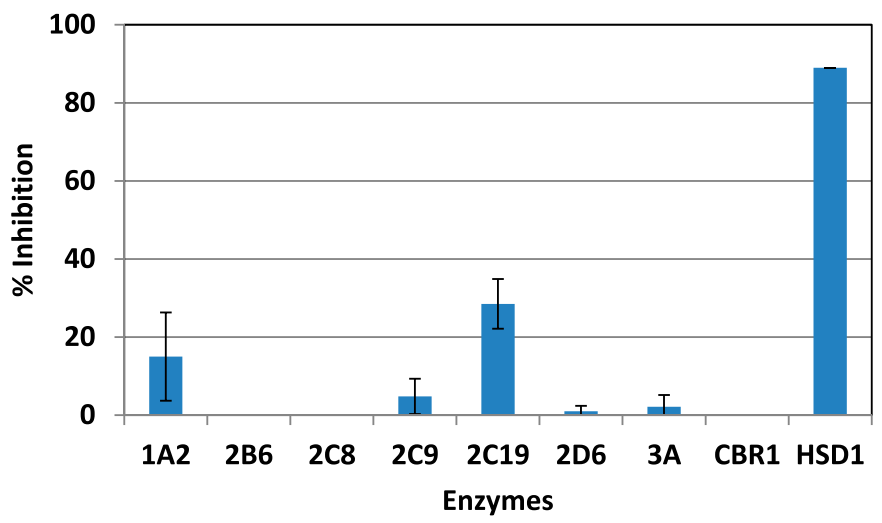

Fig. 5. $11 \beta$-HSD 1 inhibitor PF-915275 selectivity against $\mathrm{P} 450$ and CBR1 enzymes. 
TABLE 4

Percent Inhibition of doxorubicinol formation in human hepatocytes using selective $11 \beta$-HSD1 inhibitor PF-915275

\begin{tabular}{lcc}
\hline Experiments & $\begin{array}{c}\text { Cell Density } \\
\text { (Million Cells/ml) }\end{array}$ & $\begin{array}{c}\text { \% Inhibition of } \\
\text { Doxorubicinol Formation }\end{array}$ \\
\hline 1 & 2 & 29 \\
2 & 2 & 28 \\
3 & 0.5 & 27 \\
4 & 0.5 & 41 \\
5 & 2 & 19 \\
& Average and S.D. & $29 \pm 7.9$ \\
\hline
\end{tabular}

contribution of the enzyme to clearance using REF and RAF values when selective inhibitors are not available. This approach not applied to the doxorubicin case in this study owing to no significant turnover of the parent in the in vitro systems. Tissue abundance information is beneficial for development of physiologic-based pharmacokinetic models to predict human pharmacokinetics and drug-drug interaction potential.

Doxorubicin is one of the most effective chemotherapeutic agents, and it has become the "gold standard" for the treatment of various cancers, such as hematologic (lymphomas) and solid breast, ovarian, lung, and liver tumors (Hofman et al., 2015). Almost $60 \%$ of children diagnosed with cancer receive anthracyclines (e.g., doxorubicin) as part of their treatment (Völler et al., 2015); however, its effectiveness is limited by a cumulative dose-dependent cardiotoxicity that can result in irreversible heart failure (Volkova and Russell, 2011). Several mechanisms have been proposed for doxorubicin toxicity. One mechanism is that the metabolite from doxorubincin reduction (i.e., doxorubicinol) induces heart failure (Olson et al., 1988; Forrest and Gonzalez, 2000; Forrest et al., 2000; Miura et al., 2013), a mechanism that has also been shown to cause resistance in tumors (Hofman et al., 2015). The reductases involved in this biotransformation have been shown to be mediated mainly by CBR1 and AKRs (Kassner et al., 2008; Bains et al., 2010). Inhibitors of CBR 1 are currently being developed to improve the therapeutic index of doxorubincin by decreasing the formation of the doxorubicinol metabolite (Hu et al., 2015; Shi and Di, 2017). We demonstrated for the first time in this study that $11 \beta$-HSD1 is also involved in catalyzing the conversion of doxorubincin to doxorubicinol and that it is a significant pathway accounting for approximately $30 \%$ of doxorubicinol formation. The functional redundancy of reductases (CBR1, AKRs, 11 $\beta$-HSD1) provides multiple pathways to enhance the chance for elimination of the intrinsically reactive ketones and aldehydes (Shi and Di, 2017). Selective inhibitor of $11 \beta$-HSD1 PF-915275 has also been identified in this study, which enables the determination of the enzyme's contribution to metabolism. Owing to the advances and availability of the recombinant enzyme and the selective inhibitor, we were able to discover a new clearance pathway for doxorubicin, a drug which has been on the market for more than 40 years and is one of the most widely prescribed anticancer drugs.

\section{Authorship Contributions}

Participated in research design: Yang, Hua, Ryu, Yates, Chang, Zhang, Di. Conducted experiments: Yang, Hua, Ryu.

Performed data analysis: Yang, Hua, Ryu, Yates, Chang, Zhang, Di.

Wrote or contributed to the writing of the manuscript: Yang, Hua, Ryu, Yates, Zhang, Di.

\section{References}

Anderson A and Walker BR (2013) 11 $\beta$-HSD1 inhibitors for the treatment of type 2 diabetes and cardiovascular disease. Drugs 73:1385-1393.

Bains OS, Grigliatti TA, Reid RE, and Riggs KW (2010) Naturally occurring variants of human aldo-keto reductases with reduced in vitro metabolism of daunorubicin and doxorubicin. $J$ Pharmacol Exp Ther 335:533-545.

Balogh LM, Kimoto E, Chupka J, Zhang H, and Lai Y (2013) Membrane protein quantification by peptide-based mass spectrometry approaches: studies on the organic anion-transporting polypeptide family. J Proteomics Bioinform 6:229-236.

Dzyakanchuk AA, Balázs Z, Nashev LG, Amrein KE, and Odermatt A (2009) 11betaHydroxysteroid dehydrogenase 1 reductase activity is dependent on a high ratio of $\mathrm{NADPH} / \mathrm{NADP}(+)$ and is stimulated by extracellular glucose. Mol Cell Endocrinol 301: 137-141.

Forrest GL and Gonzalez B (2000) Carbonyl reductase. Chem Biol Interact 129:21-40.

Forrest GL, Gonzalez B, Tseng W, Li X, and Mann J (2000) Human carbonyl reductase overexpression in the heart advances the development of doxorubicin-induced cardiotoxicity in transgenic mice. Cancer Res 60:5158-5164.

Gronau S, Koenig Greger D, Jerg M, and Riechelmann H (2002) 11Beta-hydroxysteroid dehydrogenase 1 expression in squamous cell carcinomas of the head and neck. Clin Otolaryngol Allied Sci 27:453-457.

Hale C and Wang M (2008) Development of $11 \beta$-HSD1 inhibitors for the treatment of type 2 diabetes. Mini Rev Med Chem 8:702-710.

Hofman J, Skarka A, Havrankova J, and Wsol V (2015) Pharmacokinetic interactions of breas cancer chemotherapeutics with human doxorubicin reductases. Biochem Pharmacol 96:168-178.

Hosfield DJ, Wu Y, Skene RJ, Hilgers M, Jennings A, Snell GP, and Aertgeerts K (2005) Conformational flexibility in crystal structures of human $11 \beta$-hydroxysteroid dehydrogenase type I provide insights into glucocorticoid interconversion and enzyme regulation. J Biol Chem 280: 4639-4648.

Hu D, Miyagi N, Arai Y, Oguri H, Miura T, Nishinaka T, Terada T, Gouda H, El-Kabbani O, Xia $\mathrm{S}$, et al. (2015) Synthesis of 8-hydroxy-2-iminochromene derivatives as selective and potent inhibitors of human carbonyl reductase 1. Org Biomol Chem 13:7487-7499.

Hua W, Zhang H, Ryu S, Yang X, and Di L (2017) Human tissue distribution of carbonyl reductase 1 using proteomic approach with liquid chromatography-tandem mass spectrometry. J Pharm Sci 106: $1405-1411$.

Huuskonen P, Storvik M, Reinisalo M, Honkakoski P, Rysä J, Hakkola J, and Pasanen M (2008) Microarray analysis of the global alterations in the gene expression in the placentas from cigarette-smoking mothers. Clin Pharmacol Ther 83:542-550.

Julian LD, Wang Z, Bostick T, Caille S, Choi R, DeGraffenreid M, Di Y, He X, Hungate RW, Jaen JC, et al. (2008) Discovery of novel, potent benzamide inhibitors of $11 \beta$-hydroxysteroid dehydrogenase type 1 (11 $\beta$-HSD1) exhibiting oral activity in an enzyme inhibition ex vivo model. J Med Chem 51:3953-3960.

Kassner N, Huse K, Martin H-J, Gödtel-Armbrust U, Metzger A, Meineke I, Brockmöller J, Klein $\mathrm{K}$, Zanger UM, Maser E, et al. (2008) Carbonyl reductase 1 is a predominant doxorubicin reductase in the human liver. Drug Metab Dispos 36:2113-2120.

Malátková P and Wsól V (2014) Carbonyl reduction pathways in drug metabolism. Drug Metab Rev 46:96-123.

Miura T, Taketomi A, Nishinaka T, and Terada T (2013) Regulation of human carbonyl reductase 1 (CBR1, SDR21C1) gene by transcription factor Nrf2. Chem Biol Interact 202:126-135.

Molnari JC and Myers AL (2012) Carbonyl reduction of bupropion in human liver. Xenobiotica 42 $550-561$.

Olson RD, Mushlin PS, Brenner DE, Fleischer S, Cusack BJ, Chang BK, and Boucek RJ, Jr (1988) Doxorubicin cardiotoxicity may be caused by its metabolite, doxorubicinol. Proc Natl Acad Sci USA 85:3585-3589.

Shi SM and Di L (2017) The role of carbonyl reductase 1 in drug discovery and development. Expert Opin Drug Metab Toxicol 13:859-870.

Siu M, Johnson TO, Wang Y, Nair SK, Taylor WD, Cripps SJ, Matthews JJ, Edwards MP, Pauly TA, Ermolieff J, et al. (2009) N-(Pyridin-2-yl) arylsulfonamide inhibitors of 11betahydroxysteroid dehydrogenase type 1: discovery of PF-915275. Bioorg Med Chem Lett 19: 3493-3497.

Skarydová L and Wsól V (2012) Human microsomal carbonyl reducing enzymes in the metabolism of xenobiotics: well-known and promising members of the SDR superfamily. Drug Metab Rev 44:173-191.

Šlechtová T, Gilar M, Kalíková K, and Tesařová E (2015) Insight into trypsin miscleavage: comparison of kinetic constants of problematic peptide sequences. Anal Chem 87:7636-7643.

Volkova M and Russell R, III (2011) Anthracycline cardiotoxicity: prevalence, pathogenesis and treatment. Curr Cardiol Rev 7:214-220.

Völler S, Boos J, Krischke M, Würthwein G, Kontny NE, Boddy AV, and Hempel G (2015) Agedependent pharmacokinetics of doxorubicin in children with cancer. Clin Pharmacokinet 54: $1139-1149$.

Wang M (2006) Inhibitors of 11 beta-hydroxysteroid dehydrogenase type 1 for the treatment of metabolic syndrome. Curr Opin Investig Drugs 7:319-323.

Yang X, Atkinson K, and Di L (2016) Novel cytochrome P450 reaction phenotyping for lowclearance compounds using the hepatocyte relay method. Drug Metab Dispos 44:460-465.

Address correspondence to: Hui Zhang, Pharmacokinetics, Dynamics and Metabolism, Pfizer Inc., Eastern Point Road, Groton, CT 06340. E-mail: Hui. Zhang3@Pfizer.Com; or Li Di, Pharmacokinetics, Dynamics and Metabolism, Pfizer Inc., Eastern Point Road, Groton, CT 06340. E-mail: Li.Di@Pfizer.Com 